\title{
Waist Circumference, Body Mass Index, and Other Measures of Adiposity in Predicting Cardiovascular Disease Risk Factors among Peruvian Adults
}

\author{
K. M. Knowles, ${ }^{1}$ L. L. Paiva, ${ }^{1}$ S. E. Sanchez, ${ }^{2}$ L. Revilla, ${ }^{3}$ T. Lopez, ${ }^{3}$ M. B. Yasuda, ${ }^{4}$ \\ N. D. Yanez, ${ }^{1}$ B. Gelaye, ${ }^{1}$ and M. A. Williams ${ }^{1}$ \\ ${ }^{1}$ Department of Epidemiology, Multidisciplinary International Research Training Program, School of Public Health, \\ University of Washington, Health Sciences Building F-161E, Seattle, WA 98195, USA \\ ${ }^{2}$ Universidad San Martin de Porres and Hospital Dos de Mayo, Parque "Histoira de la Medicina Paruana" $s / n$, \\ Alt. Cdra. 13 Av. Grau-Cercado, Lima, Peru \\ ${ }^{3}$ Direccion General de Epidemiologia, Ministerio de Salud, Capac Yupanqui No. 1400 Jesus Maria, Lima 11, Peru \\ ${ }^{4}$ Laboratorio de Patologiia Clinica, Instituto Nacional de Salud del Peru, Capac Yupanqui No. 1400 Jesus Maria, Lima 11, Peru
}

Correspondence should be addressed to M. A. Williams, mawilliams84@gmail.com

Received 29 November 2010; Accepted 29 December 2010

Academic Editor: Kazuko Masuo

Copyright $\odot 2011$ K. M. Knowles et al. This is an open access article distributed under the Creative Commons Attribution License, which permits unrestricted use, distribution, and reproduction in any medium, provided the original work is properly cited.

Objectives. To examine the extent to which measures of adiposity can be used to predict selected components of metabolic syndrome (MetS) and elevated C-reactive protein (CRP). Methods. A total of 1,518 Peruvian adults were included in this study. Waist circumference (WC), body mass index (BMI), waist-hip ratio (WHR), waist-height ratio (WHtR), and visceral adiposity index (VAI) were examined. The prevalence of each MetS component was determined according to tertiles of each anthropometric measure. ROC curves were used to evaluate the extent to which measures of adiposity can predict cardiovascular risk. Results. All measures of adiposity had the strongest correlation with triglyceride concentrations (TG). For both genders, as adiposity increased, the prevalence of Mets components increased. Compared to individuals with low-BMI and low-WC, men and women with highBMI and high- WC had higher odds of elevated fasting glucose, blood pressure, TG, and reduced HDL, while only men in this category had higher odds of elevated CRP. Overall, the ROCs showed VAI, WC, and WHtR to be the best predictors for individual MetS components. Conclusions. The results of our study showed that measures of adiposity are correlated with cardiovascular risk although no single adiposity measure was identified as the best predictor for MetS.

\section{Introduction}

Worldwide, cardiovascular disease (CVD) is the primary cause of death; it killed an estimated 17.1 million people in $2004[1,2]$. Historically, CVD was thought to be a disease endemic to developed countries only [3]; however, new evidence indicates that developing countries are more strongly affected by CVD than their more affluent counterparts [1-3].

The presence of metabolic syndrome (MetS) is a major risk factor for CVD [4]. According to the third report of the national cholesterol education program (NCEP) expert panel on detection, evaluation, and treatment of high blood cholesterol in adults, MetS is defined as a co-occurrence of specific health states, including elevated triglyceride concentrations (TG), reduced high-density lipoprotein (HDL), elevated blood pressure (BP), elevated fasting glucose (FG), and high waist circumference (WC) [5]. Body mass index (BMI), WC, Waist-to-hip ratio (WHR), waist-height ratio (WHtR) [6], and visceral adiposity index (VAI) [7] have been reported as similarly predictive for the presence of MetS in men and women.

Obesity is defined by the World Health Organization (WHO) as BMI $>30 \mathrm{~kg} / \mathrm{m}^{2}$, and overweight is classified as BMI $>25 \mathrm{~kg} / \mathrm{m}^{2}[8]$. The established BMI cut-off points were designed for international use. Because of the concern that BMI cut-offs points might not accurately predict health risks 
in all populations, the WHO established a commission and charged participants with examining available data about WC and WHR [9]. In light of concerns raised about the ability of BMI alone to predict cardiovascular risk, multiple studies have recently attempted to compare BMI with WC and other anthropometric measures of obesity, such as WHR, WHtR, and VAI, as predictors for CVD risk. In a meta-analysis of abdominal obesity indices comparing BMI, WC, WHR, and WHtR, researchers concluded that $\mathrm{WHtR}$ was the best predictor for both hypertension and dyslipidemia for both men and women, while BMI was the least accurate predictor of hypertension [10]. When assessing the accuracy of VAI in comparison to BMI and WC using receiver operating characteristic (ROC) curves, Amato and colleagues found VAI to be independently associated with cardiovascular events, while BMI and WC were not found to be significant discriminators [7].

Between 2000 and 2009 among Peruvians aged 15 or older, $11.5 \%$ of men and $12.5 \%$ of women were obese [11]. A recent study in Lima found the prevalence of MetS to be $21.6 \%$ in men and $29.9 \%$ in women [12]. Given that the prevalence of obesity is on the rise, and that CVD is an important cause of morbidity and mortality in Peru, its risk factors and their measurements warrant further study for this population. The purpose of the present study was to investigate the extent to which measures of adiposity (BMI, WC, WHR, WHtR, and VAI) can be used to predict elevated C-reactive protein (CRP) and selected components of MetS: elevated TG, reduced HDL, elevated BP, and elevated FG among Peruvian adults.

\section{Materials and Methods}

The data used for the present study were gathered as part of the Prevalencia de Factores de Riesgo de Enfermedades No-Transmisibles (prevalence of risk factors for noncommunicable diseases) population-based study, known as the FRENT study. Details of the study setting, sampling, and data collection procedures have been described previously $[12,13]$. For the present analysis, we excluded participants taking antidiabetic drugs $(n=30)$, lipid lowering drugs $(n=$ $33)$, or antihypertensive drugs $(n=81)$. The final analyzed sample included 1,518 participants, 952 women $(62.7 \%)$ and 566 men $(37.3 \%)$.

Participants were interviewed by trained health professionals using a standardized instrument, previously validated by the Pan American Health Organization (PAHO) and approved by the WHO [14]. Interview questions collected consisted of socio-demographic information, smoking status, alcohol consumption, medical history, and level of physical activity. Participants' height and weight were measured in accordance with PAHO procedures with participants wearing light clothing and no shoes [14]. Waist circumference $(\mathrm{cm})$ was measured around the point halfway between the iliac crest and the sides of the lower ribs; the hip circumference $(\mathrm{cm})$ was measured using the point of maximum girth around the buttocks.

Resting mean systolic and diastolic BP were calculated as an average of two measurements: the first taken after the participant had been seated for five minutes or more, and the second measure taken 30 minutes into the interview. Blood samples were drawn from participants the day after the interview, and an individual had fasted for at least 12 hours. Aliquots of serum samples were used to determine FG, TG, HDL, and LDL concentrations using standard procedures at the Peruvian National Institute of Health Laboratory in Lima, Peru. Serum CRP concentrations were measured by an ultrasensitive competitive immunoassay (Dade Behring, Deerfield, Illinois) at the University of Washington. All laboratory procedures were conducted without knowledge of participants' medical history.

All study participants provided informed consent, and all research protocols were approved by Institutional Review Boards of National Institute of Health (Lima, Peru), Dos de Mayo Hospital (Lima, Peru), and Human Subjects Division of the University of Washington (Seattle, WA, USA).

2.1. Variable Specification. WHR was calculated as waist circumference divided by hip circumference, and WHtR was computed as waist circumference divided by height. VAI was calculated according to the definition established by Amato and colleagues [7], using VAI $=1$ as the reference for a nonobese participant with normal TG and HDL concentrations

$$
\begin{aligned}
& \text { Men: } \mathrm{VAI} \\
&=\frac{\mathrm{WC}}{39.68+(1.88 * \mathrm{BMI})} * \frac{\mathrm{TG}}{1.03} * \frac{1.31}{\mathrm{HDL}}, \\
& \text { Women: } \quad \mathrm{VAI}=\frac{\mathrm{WC}}{36.58+(1.89 * \mathrm{BMI})} * \frac{\mathrm{TG}}{0.81} * \frac{1.52}{\mathrm{HDL}} .
\end{aligned}
$$

BMI was calculated as weight $(\mathrm{kg})$ divided by height squared $\left(\mathrm{m}^{2}\right)$ and categorized using WHO guidelines (lean: $<18.5 \mathrm{~kg} / \mathrm{m}^{2}$; normal: $18.5-24.9 \mathrm{~kg} / \mathrm{m}^{2}$; overweight: $25.0-$ $29.9 \mathrm{~kg} / \mathrm{m}^{2}$; obese $\left.\geq 30 \mathrm{~kg} / \mathrm{m}^{2}\right)$. In accordance with the NCEP diagnostic criteria, MetS components were defined as (1) elevated BP (mean value of systolic blood pressure $\geq 130 \mathrm{mmHg}$, mean value of diastolic blood pressure, $\geq 85 \mathrm{mmHg}$ ); (2) abdominal obesity (waist circumference $>102 \mathrm{~cm}$ in men and $>88 \mathrm{~cm}$ in women); (3) low HDL $(<40 \mathrm{mg} / \mathrm{dL}$ in men and $<50 \mathrm{mg} / \mathrm{dL}$ in women); (4) elevated TG ( $\geq 150 \mathrm{mg} / \mathrm{dL}$ ); (5) elevated FG ( $\geq 110 \mathrm{mg} / \mathrm{dL}$ ) or current drug therapy for diabetes [5].

2.2. Statistical Analyses. Data were analyzed using Statistical Package for the Social Sciences (version 17.0, SPSS Inc., Chicago, IL, USA) software. All analyses were stratified by gender. Pearson Chi square test was used to compare socio-demographic and behavioral characteristics between men and women. Correlation between the four selected MetS components and anthropometric measurements was evaluated using Spearman's rank coefficients. Each anthropometric measurement was divided a priori into tertiles and the prevalence of each MetS component was calculated for each tertile. Categories of CRP were defined by the following tertiles: $<0.81 \mathrm{mg} / \mathrm{L}, 0.81-2.53 \mathrm{mg} / \mathrm{L}$, and $>2.53$ $\mathrm{mg} / \mathrm{L}$. Elevated CRP was defined as being in the highest tertile $(>2.53 \mathrm{mg} / \mathrm{mL})$ [13]. Logistic regression procedures 
TABLE 1: Socio-demographic characteristics of the study population.

\begin{tabular}{|c|c|c|c|}
\hline Characteristic & $\begin{array}{c}\text { Women } N=952 \\
N(\%)\end{array}$ & $\begin{array}{c}\text { Men } N=566 \\
N(\%)\end{array}$ & $P$-value \\
\hline Age (years) & & & .001 \\
\hline$\leq 24$ & $151(15.9)$ & $131(23.1)$ & \\
\hline $25-34$ & $234(24.6)$ & $146(25.8)$ & \\
\hline $35-44$ & $231(24.3)$ & $109(19.3)$ & \\
\hline $45-54$ & $173(18.2)$ & $84(14.8)$ & \\
\hline $55-64$ & $109(11.4)$ & $52(9.2)$ & \\
\hline$\geq 65$ & $54(5.7)$ & $44(7.8)$ & \\
\hline Education & & & .001 \\
\hline$\leq 6$ years & $165(17.8)$ & $59(10.7)$ & \\
\hline $7-12$ years & $429(46.4)$ & $262(47.5)$ & \\
\hline$\geq 12$ years & $331(35.8)$ & $230(41.7)$ & \\
\hline Smoking status & & & $<.001$ \\
\hline Never smoker & $800(84.0)$ & $315(55.7)$ & \\
\hline Pervious smoker & $60(6.3)$ & $69(12.2)$ & \\
\hline Current smoker & $92(9.7)$ & $182(32.2)$ & \\
\hline Currently employed & & & $<.001$ \\
\hline No & $530(55.8)$ & $194(34.4)$ & \\
\hline Yes & $419(44.2)$ & $370(65.6)$ & \\
\hline Alcohol consumption & & & $<.001$ \\
\hline Low & $572(60.1)$ & $198(35.0)$ & \\
\hline Moderate & $371(39.0)$ & $330(58.3)$ & \\
\hline Excessive & $9(0.9)$ & $38(6.7)$ & \\
\hline Body mass index $\left(\mathrm{kg} / \mathrm{m}^{2}\right)$ & & & .002 \\
\hline Underweight $(<18.5)$ & $8(0.8)$ & $10(1.8)$ & \\
\hline Normal (18.5-24.9) & $402(42.2)$ & $219(38.7)$ & \\
\hline Overweight (25.0-29.9) & $339(35.6)$ & $247(43.6)$ & \\
\hline Obesity $(\geq 30.0)$ & $203(21.3)$ & $90(15.9)$ & \\
\hline Leisure time physical activity & & & .002 \\
\hline No & $217(22.8)$ & $168(29.7)$ & \\
\hline Yes, $<150$ minutes/week & $637(66.9)$ & $328(58.0)$ & \\
\hline Yes, $\geq 150$ minutes/week & $98(10.3)$ & $70(12.4)$ & \\
\hline
\end{tabular}

*All $P$ values were obtained using Pearson Chi Square.

were used to estimate odd ratios (ORs) and 95\% confidence intervals (95\% CI) of components of MetS and CRP according to combinations of overall (BMI) and central adiposity (WC) measures. For these analyses, participants were grouped a priori as follows: low BMI and low WC (the reference group), high BMI and low WC, low BMI and high WC, and high BMI and high WC. Because there were few subjects in the low BMI and high WC category, those with low BMI and high WC were grouped with high BMI and low WC. Potential-confounding variables were selected for assessment a priori on the basis of their hypothesized relationship with adiposity measures and cardiometabolic risk. The presence of confounding was empirically assessed by entering potential covariates into a logistic regression model one at a time and by comparing the adjusted and unadjusted ORs. Final logistic regression models included covariates that altered unadjusted ORs by at least 10\% [15]. For all analyses, significance was set at a $P$ value of less than .05. Finally, receiver operating characteristic (ROC) curves with area under the curve (AUC) were used to evaluate which measure of adiposity (BMI, WC, WHR, WHtR, VAI) most accurately predicted the different components of MetS.

\section{Results}

Characteristics of study participants are summarized in Table 1. The mean age of study participants was 39.3 years (38.3 years for men and 39.9 years for women). Overall, men tended to be younger, more educated, and more likely to be employed. Men reported smoking and consuming 
TABLE 2: Spearman's rank correlation coefficients for anthropometric measurements and metabolic syndrome components.

\begin{tabular}{|c|c|c|c|c|c|}
\hline & BMI $\left(\mathrm{kg} / \mathrm{m}^{2}\right)$ & $\mathrm{WC}(\mathrm{cm})$ & WHR & WHtR & VAI \\
\hline \multicolumn{6}{|c|}{ Men } \\
\hline Fasting plasma glucose (mg/dL) & 0.330 & 0.292 & 0.205 & 0.304 & 0.222 \\
\hline Triglyceride (mg/dL) & 0.462 & 0.461 & 0.335 & 0.439 & 0.948 \\
\hline $\mathrm{HDL}(\mathrm{mg} / \mathrm{dL})$ & -0.291 & -0.286 & -0.188 & -0.247 & -0.664 \\
\hline Systolic blood pressure $(\mathrm{mmHg})$ & 0.273 & 0.301 & 0.255 & 0.291 & 0.188 \\
\hline Diastolic blood pressure $(\mathrm{mmHg})$ & 0.331 & 0.330 & 0.298 & 0.316 & 0.247 \\
\hline \multicolumn{6}{|c|}{ Women } \\
\hline Fasting plasma Glucose (mg/dL) & 0.306 & 0.301 & 0.107 & 0.301 & 0.250 \\
\hline Triglyceride (mg/dL) & 0.437 & 0.455 & 0.226 & 0.451 & 0.933 \\
\hline $\mathrm{HDL}(\mathrm{mg} / \mathrm{dL})$ & -0.220 & -0.209 & -0.194 & -0.213 & -0.618 \\
\hline Systolic blood pressure $(\mathrm{mmHg})$ & 0.296 & 0.323 & 0.180 & 0.304 & 0.271 \\
\hline Diastolic blood pressure $(\mathrm{mmHg})$ & 0.265 & 0.265 & 0.164 & 0.262 & 0.227 \\
\hline
\end{tabular}

The $P$ values for all Spearman's rank correlations listed are less than or equal to.001.

alcohol more frequently than women. On the basis of BMI values, men tended to be overweight $(43.6 \%$ versus $35.6 \%$ of women), but women were more commonly obese $(21.3 \%$ versus $15.9 \%$, resp.).

Spearman's rank correlation coefficients were used to evaluate associations between anthropometric measurements and components MetS (Table 2). The strongest correlation was between VAI with triglyceride concentrations (men: $r=0.948$, women: $r=0.933$ ), followed by VAI with HDL concentrations (men: $r=-0.664$, women: $r=$ -0.618 ); however, this was expected as TG and HDL are used to calculate VAI. For all measures of adiposity, triglyceride concentrations had the strongest positive correlation for men: BMI $(r=0.462)$, WC $(0.461)$, WHtR $(r=0.439)$, and WHR $(r=0.335)$ and for women: WC $(r=0.455)$, WHtR $(r=0.451)$, BMI $(r=0.437)$, and WHR $(r=$ 0.226). Of the measures of adiposity studied, BMI was most positively correlated with FG for both men $(r=0.330)$ and women $(r=0.306)$. Other than VAI, BMI was most strongly negatively correlated with reduced HDL.

Table 3 shows for both genders that the prevalence of elevated FG, BP, TG, and reduced HDL increased progressively as tertiles of each of the measures of adiposity studied increased. Table 4 shows the risk of MetS components in relation to central adiposity measures for men and women, adjusting for age, education, smoking, leisure time physical activity, and alcohol consumption. Compared to the low BMI \& low WC (reference group), men with high BMI or high WC had 3.40 higher odds of having elevated TG (95\% CI: 2.21-5.23), while men having both high BMI and high WC had an even higher adjusted odds ratio (AOR) (AOR: 3.89 95\% CI: 2.15-7.04). For women, these AORs were 1.72 (95\% CI: 1.02-2.91) and 4.64 (95\% CI: 3.05-7.06), respectively. Men having either high BMI or high WC had 2.04 increased odds of reduced HDL (95\% CI: 1.38-3.02), while men having high BMI and high WC had even higher odds for reduced HDL (AOR: 3.97 95\% CI: 2.20-7.18). For women, these AORs were 1.29 (95\% CI: 0.89-1.88) and 2.71 (95\% CI: 1.953.75), respectively. Men having either high BMI or high WC had 1.85 higher odds for elevated BP (95\% CI: 1.18-2.88), and men in the high BMI \& high WC category had even higher odds (AOR: 2.93, 95\% CI: 1.61-5.32). For women in the same anthropometric measures categories, these AORs were 1.45 (95\% CI: $0.82-2.58$ ) and 2.09 (95\% CI: $1.32-$ 3.32 ), respectively. Men having just one of the adiposity measurements of high BMI or high WC had 1.66 higher odds (95\% CI: 0.86-3.23) of having elevated FG, while men having both high BMI and high WC had 2.32 higher odds of having elevated FG (95\% CI: 1.03-5.19). For women with high BMI or high WC, the AOR was 1.14 (95\% CI: 0.53-2.43), and for women with both high BMI and high WC, the AOR was 2.92 (95\% CI: 1.65-5.16). Men having high BMI or high WC had 1.61 higher odds of having elevated CRP (95\% CI: 1.08-2.41), and these odds increased for men having both high BMI and high WC (AOR: 1.86 95\% CI: 1.05-3.31). For women, this association was not significant: 1.23 (95\% CI: 0.83-1.83) for those in the high BMI or high WC category, and among high BMI \& high WC category: 1.15 (95\% CI: 0.82-1.60).

Figures 1 and 2 show the level how adiposity measures predict each of the MetS components studied. As expected due to inclusion in the formula, VAI was the best predictor for elevated TG (area under curve [AUC] =0.98) among men and women $(A U C=0.97)$, and reduced HDL for men $(\mathrm{AUC}=0.82)$ and women $(\mathrm{AUC}=0.80)$. VAI and BMI were the best predictors for FG for men $(\mathrm{AUC}=0.67$ and 0.67 , resp.), while for women, WC and WHtR were the best predictors (AUC: 0.72 and 0.72, resp.). For elevated triglycerides, WC was the best predictor (AUC: 0.73) for men while for women, WHR was the best (AUC: 0.65). For elevated BP, WC was the best predictor (AUC: 0.66) for men while for women, WHtR was the best $(A U C=0.70)$. For reduced HDL, BMI was the strongest predictor for both men (AUC: 0.66) and women $(\mathrm{AUC}=0.62)$.

\section{Discussion}

To our knowledge, no research has previously been published assessing the multiple adiposity measures in predicting MetS among Peruvian adults. This study has demonstrated 
TABLE 3: Prevalence of metabolic syndrome components in relation to varying degree of adiposity as assessed using different anthropometric measures.

\begin{tabular}{|c|c|c|c|c|c|}
\hline \multirow{2}{*}{ Measurement of obesity } & & \multicolumn{4}{|c|}{ Metabolic syndrome components } \\
\hline & & $\begin{array}{c}\text { Elevated FG } \\
\%\end{array}$ & \multirow{3}{*}{$\begin{array}{c}\text { High TG } \\
\% \\
N=209\end{array}$} & \multirow{3}{*}{$\begin{array}{c}\text { Low HDL } \\
\% \\
N=252\end{array}$} & $\begin{array}{c}\text { Elevated BP } \\
\%\end{array}$ \\
\hline \multirow{2}{*}{\multicolumn{2}{|c|}{ Among Men }} & & & & \\
\hline & & & & & \\
\hline \multirow{3}{*}{$\begin{array}{l}\text { Body mass index } \\
\left(\mathrm{kg} / \mathrm{m}^{2}\right)\end{array}$} & Tertile $_{1}(<24.2)$ & 17.2 & 15.8 & 21.4 & 22.1 \\
\hline & Tertile $_{2}(23.3-27.6)$ & 28.1 & 33.0 & 36.5 & 27.9 \\
\hline & Tertile $_{3}(>27.6)$ & 54.7 & 51.2 & 42.1 & 50.0 \\
\hline \multirow{2}{*}{$\begin{array}{l}\text { Waist circumference } \\
(\mathrm{cm})\end{array}$} & Tertile $_{1}(<88.0)$ & 17.2 & 13.0 & 24.7 & 20.7 \\
\hline & Tertile $_{2}(88.0-97.0)$ & 35.9 & 38.0 & 33.9 & 29.9 \\
\hline \multirow{3}{*}{ Waist-to-hip ratio } & Tertile $_{3}(\geq 97.0)$ & 46.9 & 49.0 & 41.4 & 49.4 \\
\hline & Tertile $_{1}(<0.91)$ & 23.4 & 17.9 & 27.5 & 23.5 \\
\hline & Tertile $_{2}(0.91-0.96)$ & 31.3 & 35.7 & 33.9 & 30.7 \\
\hline \multirow[b]{2}{*}{ Waist-to-height ratio } & Tertile $_{3}(>0.96)$ & 45.3 & 46.4 & 38.6 & 45.8 \\
\hline & Tertile $_{1}(<0.52)$ & 17.2 & 13.0 & 20.3 & 20.8 \\
\hline \multirow{5}{*}{ VAI } & Tertile $_{2}(0.52-0.58)$ & 29.7 & 37.5 & 39.4 & 27.9 \\
\hline & Tertile $_{3}(\geq 0.58)$ & 53.1 & 49.5 & 40.2 & 51.3 \\
\hline & Tertile $_{1}(<2.85)$ & 17.2 & 0.0 & 11.2 & 23.4 \\
\hline & Tertile $_{2}(2.85-5.47)$ & 29.7 & 15.4 & 29.9 & 35.7 \\
\hline & Tertile $_{3}(\geq 5.47)$ & 53.1 & 84.6 & 59.0 & 40.9 \\
\hline \multicolumn{2}{|c|}{ Among women } & $N=110$ & $N=253$ & $N=575$ & $N=187$ \\
\hline \multirow{3}{*}{$\begin{array}{l}\text { Body mass index } \\
\left(\mathrm{kg} / \mathrm{m}^{2}\right)\end{array}$} & Tertile $_{1}(<24.1)$ & 16.4 & 15.0 & 26.6 & 19.8 \\
\hline & Tertile $_{2}(24.1-28.0)$ & 23.6 & 28.5 & 33.0 & 29.9 \\
\hline & Tertile $_{3}(>28.0)$ & 60.0 & 56.5 & 40.3 & 50.3 \\
\hline \multirow{3}{*}{$\begin{array}{l}\text { Waist circumference } \\
(\mathrm{cm})\end{array}$} & Tertile $_{1}(<83.0)$ & 12.7 & 10.3 & 29.3 & 14.4 \\
\hline & Tertile $_{2}(83.0-93.0)$ & 28.2 & 35.6 & 32.9 & 30.5 \\
\hline & Tertile $_{3}(\geq 93.0)$ & 59.1 & 54.2 & 37.8 & 55.1 \\
\hline \multirow[t]{3}{*}{ Waist-to-hip ratio } & Tertile $_{1}(<0.87)$ & 20.0 & 20.2 & 28.9 & 20.9 \\
\hline & Tertile $_{2}(0.87-0.92)$ & 32.7 & 30.8 & 33.6 & 36.4 \\
\hline & Tertile $_{3}(>0.92)$ & 47.3 & 49.0 & 37.5 & 42.8 \\
\hline \multirow[t]{3}{*}{ Waist-to-height ratio } & Tertile $_{1}(<0.54)$ & 11.8 & 12.3 & 27.0 & 15.5 \\
\hline & Tertile $_{2}(0.54-0.61)$ & 26.4 & 30.8 & 34.1 & 26.7 \\
\hline & Tertile $_{3}(\geq 0.61)$ & 61.8 & 56.9 & 38.9 & 57.8 \\
\hline \multirow[t]{3}{*}{ VAI } & Tertile $_{1}(<3.00)$ & 12.7 & 0.0 & 17.9 & 15.0 \\
\hline & Tertile $_{2}(3.00-5.59)$ & 21.8 & 7.9 & 32.1 & 29.9 \\
\hline & Tertile $_{3}(\geq 5.59)$ & 65.5 & 92.1 & 50.0 & 55.1 \\
\hline
\end{tabular}

FG: fasting plasma glucose; TG: triglyceride; HDL: high density lipoprotein-cholesterol; BP: blood pressure; VAI: visceral adiposity index.

the association between adiposity measures and MetS components. First, all adiposity measures were statistically significantly correlated with all MetS components studied. The prevalence of these factors increased gradually with increasing tertiles for each adiposity measure. Second, men and women with high overall and central adiposity values (i.e., high BMI \& high WC) consistently had higher odds of having cardiometabolic risk factors when compared with their leaner counterparts. Notably, elevated CRP was associated with high BMI and/or high WC for men. However, no such association was observed among women. This is in agreement with previous studies that reported a gender difference CVD risk in relation to CRP levels [13]. WC was the best measure of adiposity to predict elevated BP in men.
On the other hand, WC was most predictive of elevated FG in women.

Our observations are generally consistent with some, though not all, prior studies. Medina-Lezama et al. reported that WC was a better and accurate measure of CVD risk among Andean adults [16]. Similarly, other investigators reported that WC was a better predictor of CVD risk factors (better than BMI) among non-Hispanic black, Mexican American, and non-Hispanic white participants of the third National Health and Nutrition Examination Survey [17]. Additionally, Menke and colleagues noted that WC was a better predictor of hypertension, diabetes, low HDL cholesterol, elevated triglycerides, and insulin resistance than BMI [18]. Our findings and those of others [16-18] are somewhat 
TABLE 4: Risk of metabolic syndrome components in relation to visceral adiposity.

\begin{tabular}{|c|c|c|c|c|c|}
\hline & \multirow{2}{*}{$\begin{array}{c}\text { Low BMI \& Low WC } \\
\text { OR (CI) }\end{array}$} & \multicolumn{2}{|c|}{ High BMI or High $\mathrm{WC}^{* *}$} & \multicolumn{2}{|c|}{ High BMI and High WC } \\
\hline & & $\mathrm{OR}(\mathrm{CI})$ & $P$ value $(\mathrm{SE})$ & $\mathrm{OR}(\mathrm{CI})$ & $P$ value (SE) \\
\hline \multicolumn{6}{|c|}{ Among Women } \\
\hline Elevated triglyceride & 1.00 (Reference) & $2.44(1.50-3.98)$ & $0.000(0.249)$ & $6.47(4.37-9.58)$ & $0.000(0.200)$ \\
\hline Adjusted* & 1.00 (Reference) & $1.72(1.02-2.91)$ & $0.042(0.268)$ & $4.64(3.05-7.06)$ & $0.000(0.214)$ \\
\hline Reduced HDL & 1.00 (Reference) & $1.28(0.89-1.83)$ & $0.184(0.183)$ & $2.64(1.96-3.56)$ & $0.000(0.152)$ \\
\hline Adjusted* & 1.00 (Reference) & $1.29(0.89-1.88)$ & $0.178(0.191)$ & $2.71(1.95-3.75)$ & $0.000(0.167)$ \\
\hline Elevated BP & 1.00 (Reference) & $1.99(1.20-3.30)$ & $0.007(0.258)$ & $3.40(2.27-5.08)$ & $0.000(0.205)$ \\
\hline Adjusted* & 1.00 (Reference) & $1.45(0.82-2.58)$ & $0.206(0.294)$ & $2.09(1.32-3.32)$ & $0.002(0.236)$ \\
\hline Elevated fasting glucose & 1.00 (Reference) & $1.70(0.83-3.45)$ & $0.144(0.362)$ & $4.34(2.54-7.41)$ & $0.000(0.273)$ \\
\hline Adjusted* & 1.00 (Reference) & $1.14(0.53-2.43)$ & $0.744(0.389)$ & $2.92(1.65-5.16)$ & $0.000(0.291)$ \\
\hline Elevated CRP & 1.00 (Reference) & $1.46(1.00-2.14)$ & $0.049(0.193)$ & $1.29(0.95-1.76)$ & $0.102(0.157)$ \\
\hline Adjusted* & 1.00 (Reference) & $1.23(0.83-1.83)$ & $0.310(0.203)$ & $1.15(0.82-1.60)$ & $0.429(0.171)$ \\
\hline \multicolumn{6}{|c|}{ Among Men } \\
\hline Elevated Triglyceride & 1.00 (Reference) & $3.81(2.53-5.75)$ & $0.000(0.209)$ & $5.65(3.20-9.96)$ & $0.000(0.289)$ \\
\hline Adjusted* & 1.00 (Reference) & $3.40(2.21-5.23)$ & $0.000(0.220)$ & $3.89(2.15-7.04)$ & $0.000(0.303)$ \\
\hline Reduced HDL & 1.00 (Reference) & $2.21(1.53-3.20)$ & $0.000(0.189)$ & $4.33(2.48-7.57)$ & $0.000(0.284)$ \\
\hline Adjusted* & 1.00 (Reference) & $2.04(1.38-3.02)$ & $0.000(0.199)$ & $3.97(2.20-7.18)$ & $0.000(0.302)$ \\
\hline Elevated BP & 1.00 (Reference) & $1.98(1.29-3.04)$ & $0.002(0.218)$ & $3.67(2.08-6.49)$ & $0.000(0.291)$ \\
\hline Adjusted* & 1.00 (Reference) & $1.85(1.18-2.88)$ & $0.007(0.227)$ & $2.93(1.61-5.32)$ & $0.000(0.305)$ \\
\hline Elevated fasting glucose & 1.00 (Reference) & $2.10(1.11-3.96)$ & $0.022(0.324)$ & $3.61(1.67-7.81)$ & $0.001(0.394)$ \\
\hline Adjusted* & 1.00 (Reference) & $1.66(0.86-3.23)$ & $0.135(0.339)$ & $2.32(1.03-5.19)$ & $0.041(0.412)$ \\
\hline Elevated CRP & 1.00 (Reference) & $1.66(1.13-2.43)$ & $0.010(0.195)$ & $2.04(1.18-3.52)$ & $0.010(0.278)$ \\
\hline Adjusted* & 1.00 (Reference) & $1.61(1.08-2.41)$ & $0.019(0.204)$ & $1.86(1.05-3.31)$ & $0.034(0.293)$ \\
\hline
\end{tabular}

* Adjusted for age, education, smoking, leisure time physical activity, and alcohol consumption.

** Low BMI and High WC combined with High BMI and Low WC.

inconsistent with other reports. For instance, Wildman and colleagues reported that WC and BMI were equally predictive of CVD risk [19]. Moreover, results from a 2007 metaanalysis [20] suggested that measures of overall obesity (BMI) and measures of central obesity (WHR and WC) performed equally well in predicting incident type 2 diabetes. Other investigators, however, have reported that WHtR is the best predictor of CVD risk and other cardiometabolic risk factors (including hypertension and dyslipidemia) than other anthropometric measurements [19-26]. In a metaanalysis of indices of abdominal obesity, Lee et al. reported that BMI was the poorest discriminator, whilst WHtR was the best discriminator for hypertension, diabetes, and dyslipidemia for both men and women [10]. Herrera and colleagues also reported that WHtR was the most accurate measure of coronary heart disease risk, followed by WC, and BMI, in their study [22]. Finally, some have suggested that WHR, because it takes body fat distribution into account by showing abdominal and peripheral adiposity, may be the ideal measurement of adiposity [27]. However, we found WHR to have the weakest correlation and lowest AUC values of the adiposity measures for all MetS components, with the exception of elevated BP in men. VAI appeared to be the best predictor of elevated TG and low HDL in our study. However, it is important to note that triglyceride and HDL concentrations are included in the calculation of VAI values. Our observation of higher odds of cardiometabolic risks among men and women with combined high overall adiposity and central adiposity (i.e., high BM and WC values) is biologically plausible, as intra-abdominal fat is known to be highly associated with all components of MetS [28].

As noted by Paniagua et al. [26], heterogeneity in study findings across studies that have assessed cardiometabolic risk factors in relation to indices of adiposity may be attributable to differences in race/ethnicity, age, and gender distributions of participants across study populations. A number of investigators have reported differences in the predictive value of obesity indicators according to ethnicity $[29,30]$. Vazquez et al. noted that central obesity was a stronger predictor of incident type 2 diabetes than were measures of total body fat [20]. However, measures of overall obesity were better predictor of type 2 diabetes in US and European Caucasian [31]. Though no anthropometric measurement was consistently the best predictor for MetS among the present population of Peruvian adults, we noted that VAI, WC, and WHtR to be the best predictors for individual MetS components.

Strengths of our study include the extensive CVD riskfactor data available for study participants and the unique opportunity to assess these risk factors in a population-based sample representative of adult residents of Lima and Callao, Peru. Limitations of our study include the cross-sectional design which did not allow us to assess the temporality of the relation between the adiposity measures and metabolic 


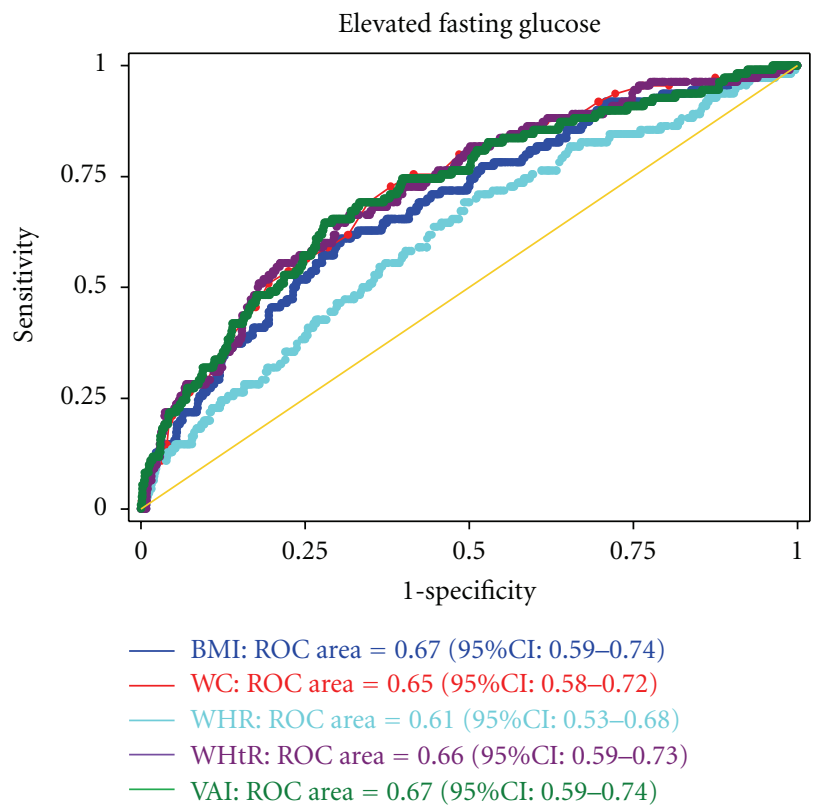

(a)

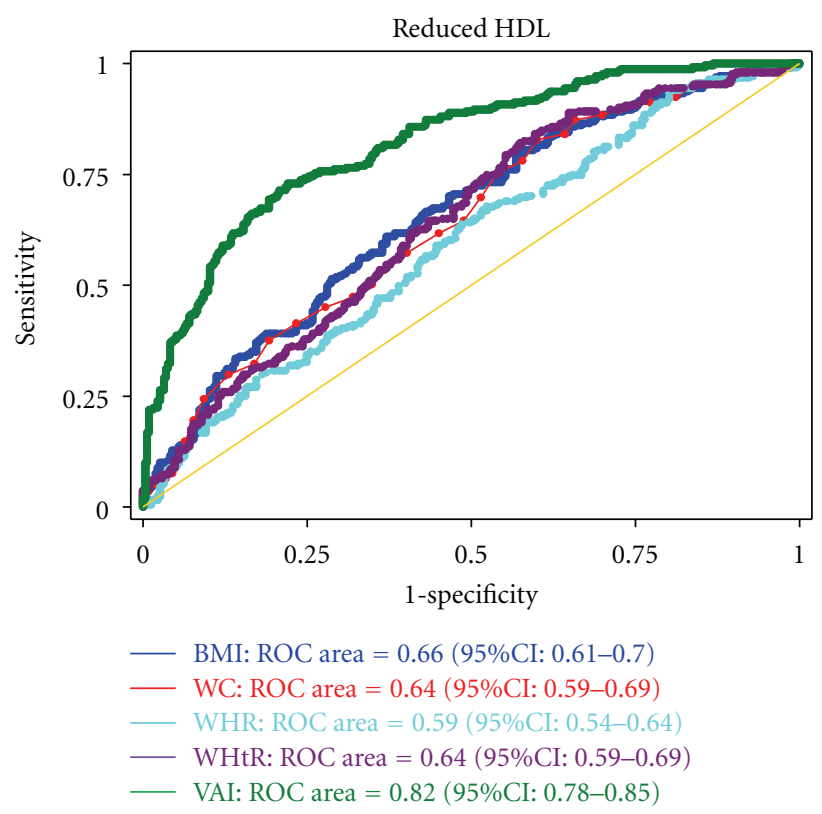

(c)

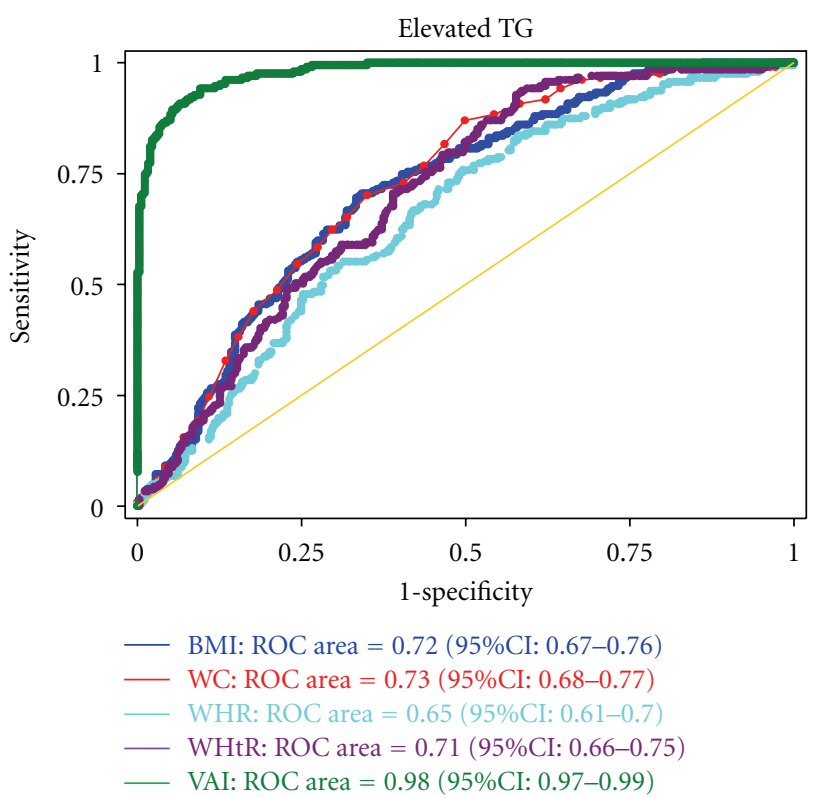

(b)

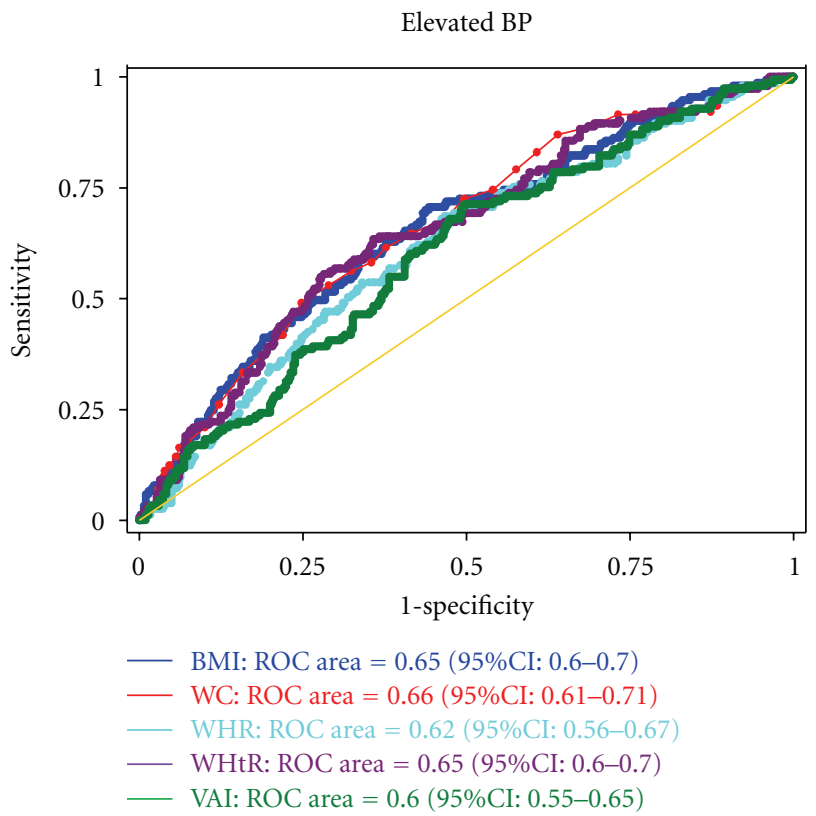

(d)

FIGURE 1: Receiver operating characteristic (ROC) curves with area under curve (AUC) and 95\% confidence intervals of body mass index (BMI), waist circumference (WC), waist-to-hip ratio (WHR) and waist-to-height ratio (WHtR) for predicting cardiovascular disease risk factors among Peruvian men.

syndrome components. Some nonsystematic error in reporting of smoking history, physical activity, and other covariates may have occurred. Additionally, despite adjustment for multiple confounders, residual confounding by unmeasured or imprecisely measured covariates may persist. Finally, concordance of our study results with previous reports from geographically, racially, and ethnically diverse populations, in part, attenuates these concerns.
Although the best adiposity measurement for predicting CVD remains controversial, in our study most measures of adiposity were correlated with the cardiometabolic factors of interest. The results of our study underscore the importance of using simple, broadly applicable measures of adiposity such as WC and WHtR in community-based epidemiologic studies. These relatively inexpensive and easily obtained measures are useful for assessing cardiovascular 


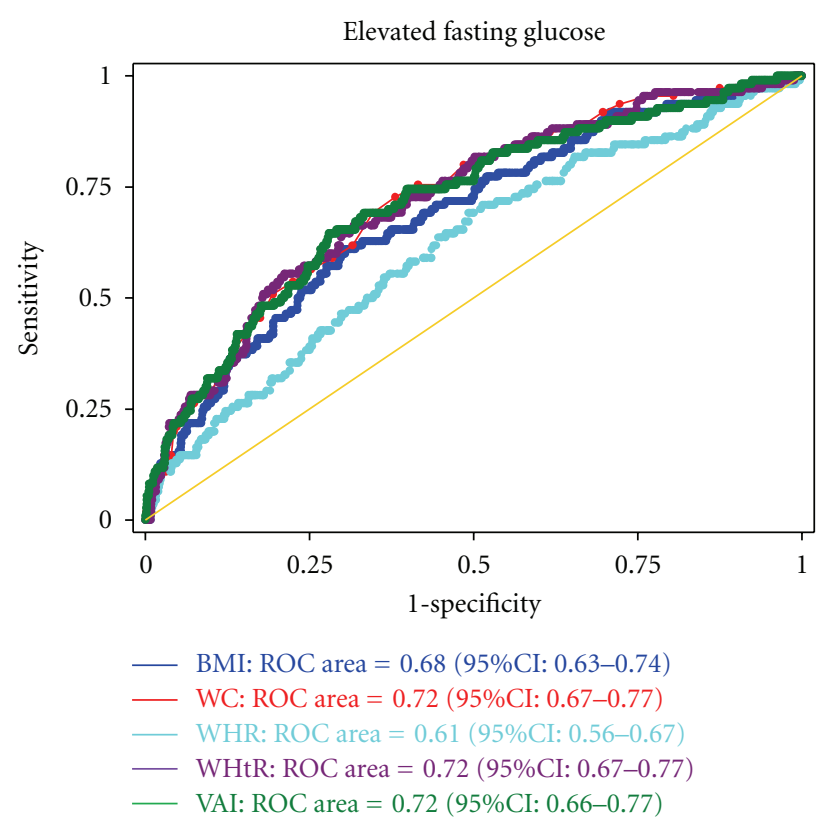

(a)

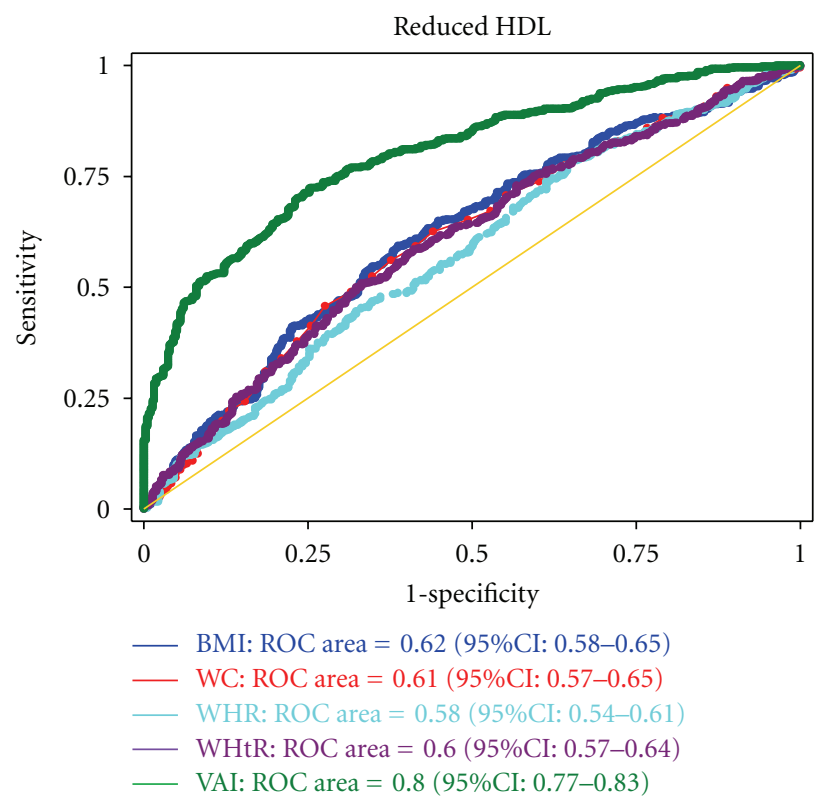

(c)

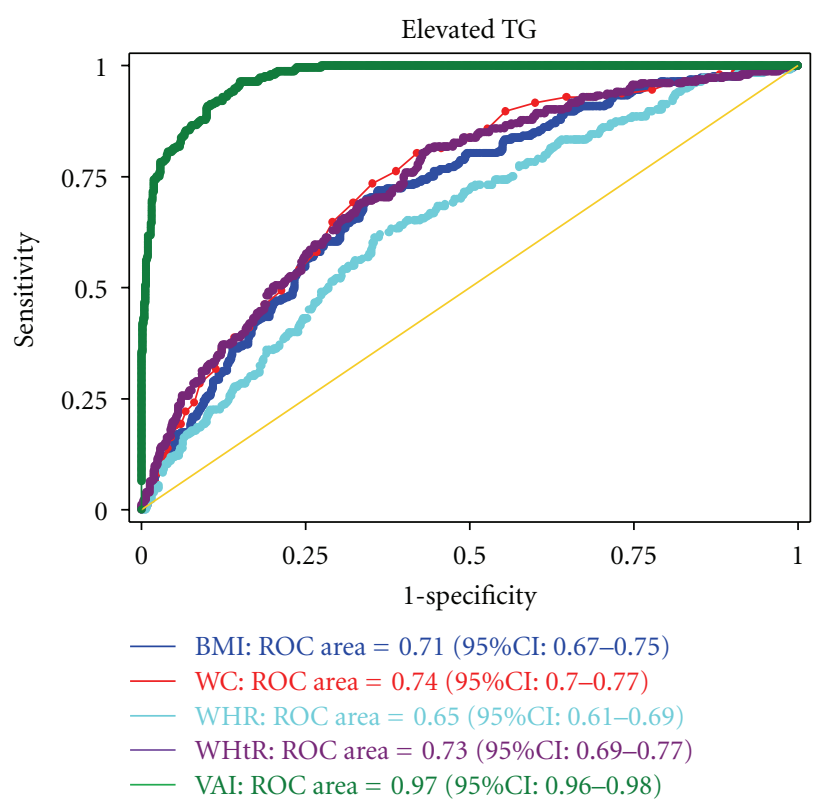

(b)

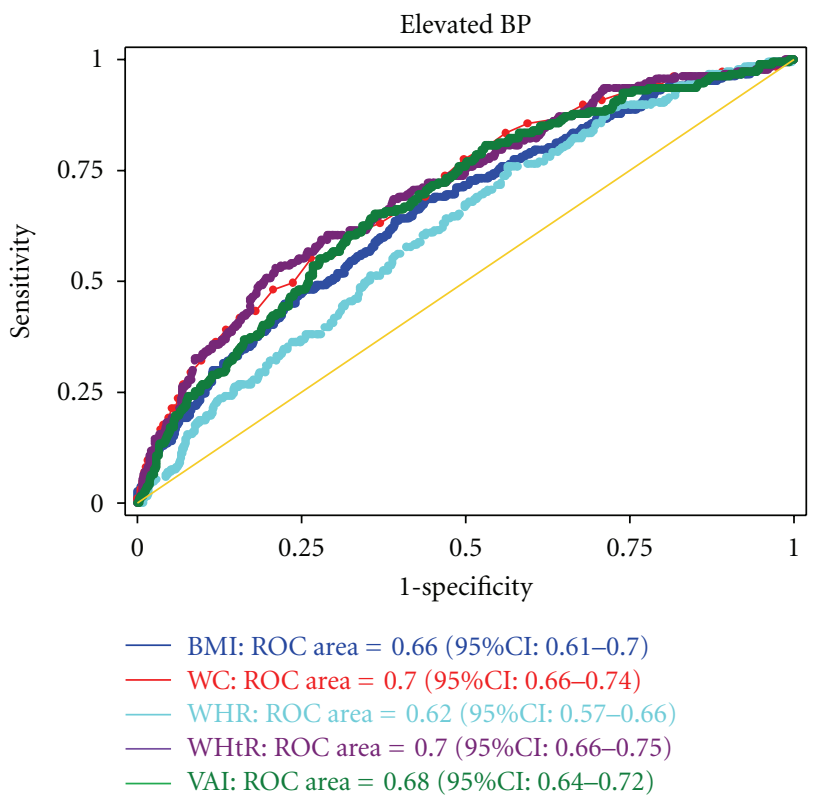

(d)

FIGURE 2: Receiver operating characteristic (ROC) curves with area under curve (AUC) and 95\% confidence intervals of body mass index (BMI), waist circumference (WC), waist-to-hip ratio (WHR), and waist-to-height ratio (WHtR) for predicting cardiovascular disease risk factors among Peruvian Women.

disease risk in nonclinical settings. Though the various measurements each have advantages and disadvantages, it is evident that, to date, no single measurement can be identified as the optimal choice for CVD prediction on its own. To this effect, the United States National Institutes of Health $(\mathrm{NIH})$ now recommends the use of WC in conjunction with BMI as a complementary indicator of health risk among normal and overweigh subjects [32]. The overall results of our study showed that measures of adiposity are correlated with cardiovascular risk among Peruvian adults. Investigators in Latin America have called for a country-specific epidemiological data to help bring public health policy changes for surveillance, prevention, and intervention [33]. The high prevalence of MetS, obesity, and observed associations of cardiometabolic risks with adiposity measures reported in this study calls for increased efforts aimed towards clinical preventive services to identify and control the existing metabolic abnormalities among 
patients. Additionally, development and implementation of public health programs that promote healthful behaviors including increased physical activity, eating balanced diets, and avoidance of adult weight gain are needed to help reduce the burden of noncommunicable diseases among Peruvian adults.

\section{Conflict of Interests}

The authors declare that they have no conflict of interests.

\section{Authors' Contributions}

L. Revilla, TTC, M. B. Yasuda, and S. E. Sanchez participated in the design of the study and carried out data collection. K. M. Knowles and L. L. Paiva participated in statistical analysis, interpretation of results, and drafting the paper. S. E. Sanchez and N. D. Yanez led the analysis, supervised research trainees, and participated in interpretation of results and in drafting the paper. B. Gelaye and M. A. Williams conceived and participated in data analysis, interpretation of results, and providing critical review of the paper. All authors read and approved the final paper. K. M. Knowles and L. L. Paiva contributed equally to this work.

\section{Acknowledgments}

The FRENT study was supported by General Direction of Epidemiology Bureau (Direccion Nacional de Epidemiologia) and the Peruvian National Institute of Health, Lima, Peru. The present analysis was completed while Ms Kaylee Knowles and Ms Laura Paiva were research training fellows with the Multidisciplinary International Research Training (MIRT) Program of the School of Public Health, University of Washington. The MIRT Program is supported by an Award from the National Institutes of Health, National Center on Minority Health and Health Disparities (no. T37MD001449).

\section{References}

[1] WHO, The Global Burden of Disease 2004 Update, WHO, Geneva, Switzerland, 2004.

[2] WHO, "World Health Report. Prevention Chronic Disease: A vital Investment," Geneva, Switzerland, 2008, http://www. who.int/chp/chronic_disease_report/contents/part1.pdf.

[3] M. Ezzati, S. Vander Hoorn, C. M. M. Lawes et al., "Rethinking the "diseases of affluence" paradigm: global patterns of nutritional risks in relation to economic development," PLoS Medicine, vol. 2, no. 5, article e133, 2005.

[4] E. S. Ford, "Risks for all-cause mortality, cardiovascular disease, and diabetes associated with the metabolic syndrome: a summary of the evidence," Diabetes Care, vol. 28, no. 7, pp. 1769-1778, 2005.

[5] S. M. Grundy, D. Becker, L. T. Clark et al., "Detection, evaluation, and treatment of high blood cholesterol in adults (Adult Treatment Panel III)," Circulation, vol. 106, no. 25, pp. 3143-3421, 2002.

[6] A. Bosy-Westphal, C. Geisler, S. Onur et al., "Value of body fat mass vs anthropometric obesity indices in the assessment of metabolic risk factors," International Journal of Obesity, vol. 30, no. 3, pp. 475-483, 2006.

[7] M. C. Amato, C. Giordano, M. Galia et al., "Visceral adiposity index: a reliable indicator of visceral fat function associated with cardiometabolic risk," Diabetes Care, vol. 33, no. 4, pp. 920-922, 2010.

[8] WHO, Obesity: Preventing and Managing the Global Epidemic, Geneva, Switzerland, 2000.

[9] C. Nishida, G. T. Ko, and S. Kumanyika, "Body fat distribution and noncommunicable diseases in populations: overview of the 2008 WHO Expert Consultation on Waist Circumference and Waist-Hip Ratio," European Journal of Clinical Nutrition, vol. 64, no. 1, pp. 2-5, 2010.

[10] C. M.Y. Lee, R. R. Huxley, R. P. Wildman, and M. Woodward, "Indices of abdominal obesity are better discriminators of cardiovascular risk factors than BMI: a meta-analysis," Journal of Clinical Epidemiology, vol. 61, no. 7, pp. 646-653, 2008.

[11] WHO, World Health Statistics, WHO, Geneva, Switzerland, 2010.

[12] B. Gelaye, L. Revilla, T. Lopez, S. Sanchez, and M. A. Williams, "Prevalence of metabolic syndrome and its relationship with leisure time physical activity among Peruvian adults," European Journal of Clinical Investigation, vol. 39, no. 10, pp. 891$898,2009$.

[13] B. Gelaye, L. Revilla, T. Lopez et al., "Association between insulin resistance and c-reactive protein among Peruvian adults," Diabetology and Metabolic Syndrome, vol. 2, no. 1, article 30, 2010.

[14] PAHO: PAN AMERICAN HEALTH ORGANIZATION, "Countrywide Integrated Noncommunicable Diseases Intervention (CINDI) Programme," September 2010, http://www. paho.org/english/ad/dpc/nc/hcncindi.pdf.

[15] K. J. Rothman and S. Greenland, Modern Epidemiology, Lippincott-Raven, Philadelphia, Pa, USA, 1998.

[16] J. Medina-Lezama, C. A. Pastorius, H. Zea-Diaz et al., "Optimal definitions for abdominal obesity and the metabolic syndrome in Andean Hispanics: the PREVENCION study," Diabetes Care, vol. 33, no. 6, pp. 1385-1388, 2010.

[17] S. Zhu, S. B. Heymsfield, H. Toyoshima, Z. Wang, A. Pietrobelli, and S. Heshka, "Race-ethnicity-specific waist circumference cutoffs for identifying cardiovascular disease risk factors," American Journal of Clinical Nutrition, vol. 81, no. 2, pp. 409-415, 2005.

[18] A. Menke, P. Muntner, R. P. Wildman, K. Reynolds, and HE. Jiang, "Measures of adiposity and cardiovascular disease risk factors," Obesity, vol. 15, no. 3, pp. 785-795, 2007.

[19] R. P. Wildman, D. Gu, K. Reynolds, X. Duan, X. Wu, and J. He, "Are waist circumference and body mass index independently associated with cardiovascular disease risk in Chinese adults?" American Journal of Clinical Nutrition, vol. 82, no. 6, pp. 11951202, 2005.

[20] G. Vazquez, S. Duval, D. R. Jacobs, and K. Silventoinen, "Comparison of body mass index, waist circumference, and waist/hip ratio in predicting incident diabetes: a metaanalysis," Epidemiologic Reviews, vol. 29, no. 1, pp. 115-128, 2007.

[21] W. Aekplakorn, V. Pakpeankitwatana, C. M. Y. Lee et al., "Abdominal obesity and coronary heart disease in Thai men," Obesity, vol. 15, no. 4, pp. 1036-1042, 2007.

[22] V. M. Herrera, J. P. Casas, J. J. Miranda et al., "Interethnic differences in the accuracy of anthropometric indicators of obesity in screening for high risk of coronary heart disease," International Journal of Obesity, vol. 33, no. 5, pp. 568-576, 2009. 
[23] S. Y. Ho, T. H. Lam, and E. D. Janus, "Waist to stature ratio is more strongly associated with cardiovascular risk factors than other simple anthropometric indices," Annals of Epidemiology, vol. 13, no. 10, pp. 683-691, 2003.

[24] W.-Y. Lin, L.-T. Lee, C.-Y. Chen et al., "Optimal cut-off values for obesity: using simple anthropometric indices to predict cardiovascular risk factors in Taiwan," International Journal of Obesity, vol. 26, no. 9, pp. 1232-1238, 2002.

[25] M. A. Sayeed, H. Mahtab, Z. A. Latif et al., "Waist-to-height ratio is a better obesity index than body mass index and waist-to-hip ratio for predicting diabetes, hypertension and lipidemia," Bangladesh Medical Research Council Bulletin, vol. 29, no. 1, pp. 1-10, 2003.

[26] L. Paniagua, V. Lohsoonthorn, S. Lertmaharit, W. Jiamjarasrangsi, and M. A. Williams, "Comparison of waist circumference, body mass index, percent body fat and other measure of adiposity in identifying cardiovascular disease risks among Thai adults," Obesity Research \& Clinical Practice, vol. 2, no. 3, pp. 215-223, 2008.

[27] D. Canoy, "Distribution of body fat and risk of coronary heart disease in men and women," Current Opinion in Cardiology, vol. 23, no. 6, pp. 591-598, 2008.

[28] D. B. Carr, K. M. Utzschneider, R. L. Hull et al., "Intraabdominal fat is a major determinant of the National Cholesterol Education Program Adult Treatment Panel III criteria for the metabolic syndrome," Diabetes, vol. 53, no. 8, pp. 20872094, 2004.

[29] P. Deurenberg, M. Yap, and W. A. Van Staveren, "Body mass index and percent body fat: a meta analysis among different ethnic groups," International Journal of Obesity, vol. 22, no. 12, pp. 1164-1171, 1998.

[30] B. B. Duncan, L. E. Chambless, M. I. Schmidt et al., "Correlates of body fat distribution: variation across categories of race, sex, and body mass in the atherosclerosis risk in communities study," Annals of Epidemiology, vol. 5, no. 3, pp. 192-200, 1995.

[31] J. M. Chan, E. B. Rimm, G. A. Colditz, M. J. Stampfer, and W. C. Willett, "Obesity, fat distribution, and weight gain as risk factors for clinical diabetes in men," Diabetes Care, vol. 17, no. 9, pp. 961-969, 1994.

[32] NIH, The Practical Guide to the Identification, Evaluation and Treatment of Overweight and Obesity in Adults, National Institutes of Health, Bethesda, Md, USA, 2000.

[33] H. Schargrodsky, R. Hernández-Hernández, B. M. Champagne et al., "CARMELA: assessment of cardiovascular risk in seven Latin American cities," American Journal of Medicine, vol. 121, no. 1, pp. 58-65, 2008. 


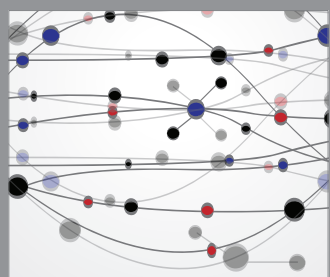

The Scientific World Journal
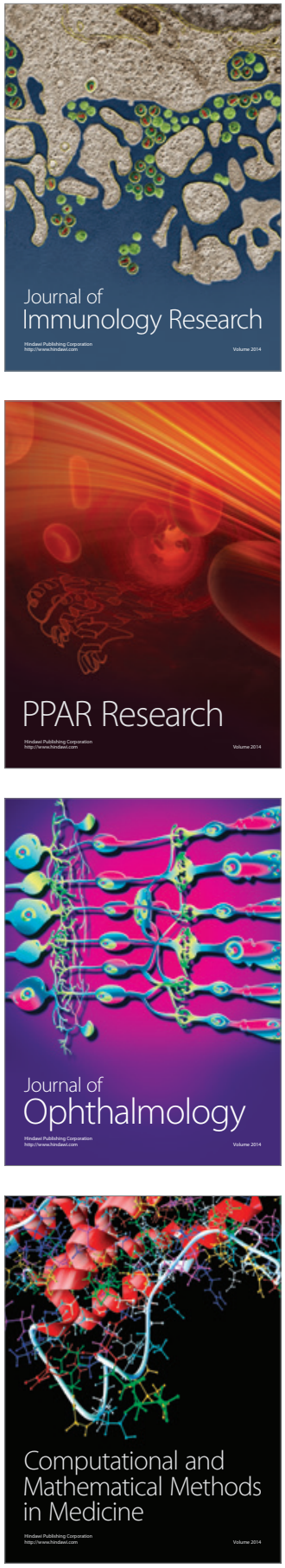

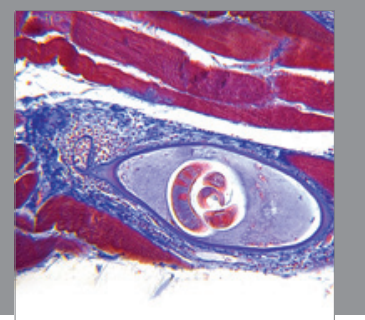

Gastroenterology

Research and Practice
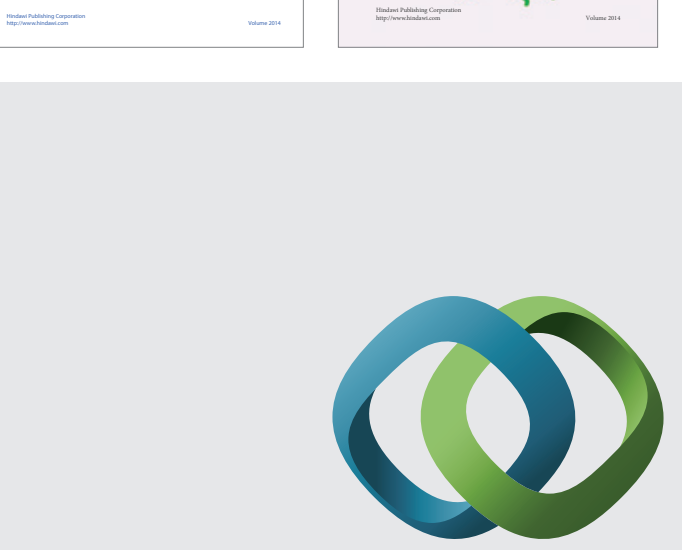

\section{Hindawi}

Submit your manuscripts at

http://www.hindawi.com
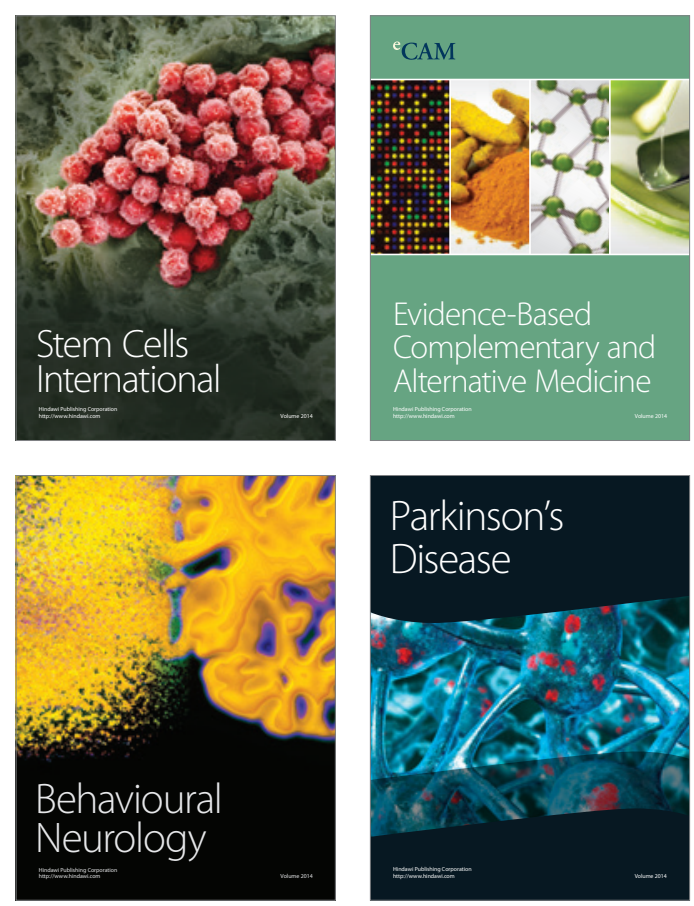

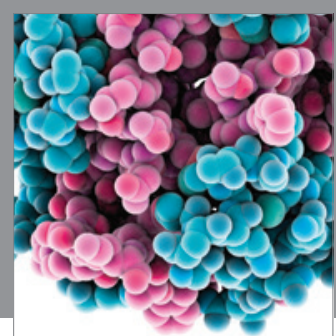

Journal of
Diabetes Research

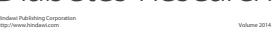

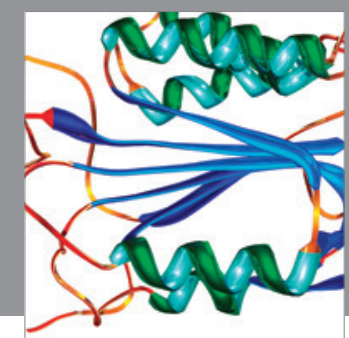

Disease Markers
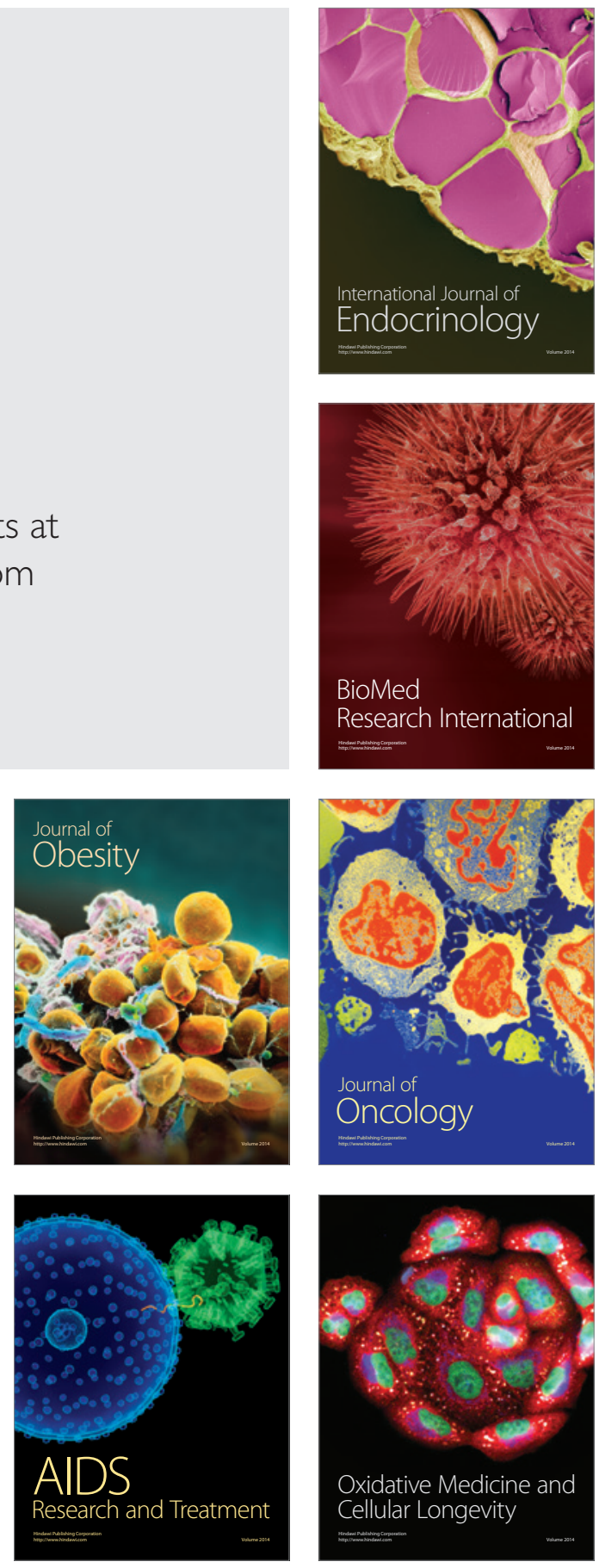